\title{
Nice to Know: Impact of NICE Guidelines on Ketogenic Diet Services Nationwide.
}

\author{
V.J. Whiteley ${ }^{1,2}$, K.J. Martin-McGill ${ }^{3,4}$, J.H. Carroll ${ }^{4}$, H. Taylor ${ }^{5}$, N.E. Schoeler ${ }^{6}$ on \\ behalf of The Ketogenic Dietitians Research Network (KDRN).
}

1. Therapy and Dietetics, Royal Manchester Children's Hospital, Manchester, UK

2. School of Health \& Society, University of Salford, Greater Manchester, UK

3. Clinical sciences and nutrition, University of Chester, Chester, UK

4. Institute of Translational Medicine, University of Liverpool, Liverpool, UK

5. Institute of Health and Community, School of Health Professions, University of Plymouth, Plymouth, UK

6. Dietetics, Sheffield Children's Hospital, Sheffield, UK

7. Clinical Neurosciences, UCL Great Ormond Street Institute of Child Health, London, UK

\section{Correspondence}

V.J. Whiteley, Therapy and Dietetics, Royal Manchester Children's Hospital, Oxford

Road, Manchester, M13 9WL. UK.

Email: Victoria.whiteley@mft.nhs.uk

Tel: +441617017580

\section{Kirsty.martin@liverpool.ac.uk}

Jennifer.carroll@plymouth.ac.uk

Hannah.taylor2@sch.nhs.uk

N.schoeler@ucl.ac.uk

Keywords: Ketogenic diet, Epilepsy, Ketogenic diet services.

\section{Acknowledgements}

We thank Matthew's Friends and The Daisy Garland for advertising the survey, Vitaflo (International) Ltd and Nutricia Advanced Medical Nutrition for supporting KDRN meetings and finally the members of the Ketogenic Dietitians Research 
Network (KDRN) for their support and expertise including the participating centres; Addenbrookes Hospital, Alder Hey Children's Hospital, Birmingham Children's Hospital, Bon Secours Hospital, Bradford Teaching Hospitals, Bristol Royal Hospital for Children, Cork University Hospital, David Lewis centre, Evelina Children's Hospital, Great North Children's Hospital, Great Ormond Street Hospital, Leeds Children's Hospital, John Radcliffe Hospital, Leicester Royal Infirmary, Matthews Friends Lingfield, National Hospital for Neurology and Neurosurgery, Nottingham University Hospitals, Nutrikids Dublin and Longford, Our Lady's Children's Hospital Crumlin, Raigmore Hospital, Royal Belfast Hospital for Sick Children, Royal Derby Hospital, Royal Devon and Exeter Hospital, Royal Gloucester Hospital, Royal Hospital for Sick Children Edinburgh, Royal Hospital for Sick Children Glasgow, Royal Manchester Children's Hospital, Royal Preston Hospital, Sheffield Children's Hospital, Southampton General Hospital, Southmead Hospital, St George's Hospital, St. James's Hospital, Tayside Children's Hospital, Temple Street Children's University Hospital, The Barberry Centre, The Walton Centre NHS Foundation Trust.

\section{Nice to Know: Impact of NICE Guidelines on Ketogenic Diet Services Nationwide.}

\section{Abstract}

Background: In 2012, National Institute for Health and Care Excellence (NICE) Clinical Guidelines for Epilepsies: Diagnosis and Management (CG137) included, for the first time, ketogenic diets (KDs) as a treatment option for drug resistant paediatric epilepsy. The recommendation was made to refer children and young people with epilepsy whose seizures have not responded to appropriate anti-epileptic drugs to a tertiary paediatric epilepsy specialist for consideration of the use of KDs. We aimed to assess the impact of this change in guidance on numbers of ketogenic centres and patients following KDs for epilepsy in the UK and Ireland.

Methods: An online survey was circulated to ketogenic dietitians from the UK and Ireland. Results were compared with similar surveys published in 2000 and 2010.

Results: The number of centres offering KDs for treatment of epilepsy has risen 
from 22 in 2000, to 28 in 2010 to 39 in 2017 (77\% overall increase). Seven of these centres accept adult referrals, in comparison to only two centres in 2010. Patient numbers have increased from 101 in 2000 to 754 in 2017. 267 patients are waiting to commence KD at 31 centres.

Conclusions: Over the last seven years, the number of patients treated with a KD for epilepsy in the UK and Ireland has increased by $647 \%$, with a $77 \%$ increase in the number of centres offering KDs. Despite this rapid growth, there is ongoing demand for patients to be considered for dietary therapy, highlighting the need for continued expansion of KD services nationally.

\section{Introduction}

Ketogenic diets (KDs) are high-fat, restricted carbohydrate diets that have been used as a treatment for drug-resistant epilepsy since the 1920 s $^{(1)}$. There are different types of KDs in use: the classical KD (CKD) ${ }^{(2)}$, medium chain triglyceride (MCT) KD ${ }^{(3)}$, Modified Atkins diet (MAD) ${ }^{(4)}$ modified ketogenic diet (MKD) ${ }^{(5,6)}$ and the low glycaemic index treatment (LGIT) ${ }^{(7)}$. The MAD, MKD and LGIT are less restrictive versions of a $\mathrm{KD}$, often using household measures rather than accurate weighing of foods, and greater freedom in consumption of protein and, in some cases, carbohydrate. This more liberal approach may help improve tolerability and adherence ${ }^{(8)}$, but the principles of a high-fat, low-carbohydrate diet remain, requiring significant dietary adjustment for the child, young person or adult undertaking KD therapy.

KDs are a well-established non-pharmacological treatment for drug resistant epilepsy evidenced by an increasing number of randomised controlled trials (RCTs) in the paediatric population. The most recent Cochrane review $(2018)^{(8)}$ concluded that evidence from 10 trials showed promising results for the use of KDs in epilepsy treatment. $\geq 50 \%$ seizure reduction was achieved in $38-85 \%(9,10)$ of participants treated with classical $4: 1 \mathrm{KD}$ and $42-60 \%{ }^{(11,12)}$ treated with MAD for a duration of 3 months. Seizure freedom varies with $1-55 \%(9,10)$ of participants following a classical $\mathrm{KD}$ and $10-25 \%{ }^{(11,12)}$ following a MAD for 3 months achieving this. 
KDs are being increasingly used for adults with epilepsy and both the classical KD and MAD show promise in terms of feasibility and tolerability (13-15). Effectiveness was recently assessed in two RCTs comparing MAD to control (treatment as usual). Zare et al. (16) reported $35.3 \%$ of participants to be responders achieving $>50 \%$ reduction in seizure frequency after 2 months treatment with MAD. In contrast, Kverneland et al. (17) found only $12.5 \%$ of participants experienced $>50 \%$ seizure reduction after 3 months treatment with MAD. However, this finding was not statistically significant. At best, they report a significant reduction in diet versus control group for moderate benefit only (25-50\% seizure reduction). Low participant numbers, epilepsy type (focal versus generalised) and length of time with drug resistant epilepsy are possible reasons for difference in findings. Further, large scale $\mathrm{RCTs}$ are required in this area.

The use of KDs to treat childhood epilepsy in the UK was examined in $2000{ }^{(18)}$ and then again in $2010{ }^{(19)}$ via a survey to paediatric ketogenic dietitians. The number of centres offering KDs and patients following a KD increased in this time period. These surveys were undertaken prior to 2012 and the publication of the National Institute for Health and Care Excellence (NICE) guidance on the diagnosis and management of epilepsies (CG137; 2012) ${ }^{20)}$ which, for the first time, recommended consideration of a KD for children and young people with drug resistant epilepsy. One may expect that referrals for KD therapy and demand on KD services would have increased further since the change in NICE guidance ${ }^{(20)}$. This survey aimed to reassess the use of KDs aiming to assess the changes in practice and service provision since 2007.

\section{Methods}

An online survey was devised by a group of adult and paediatric ketogenic dietitians, as part of the Ketogenic Dietitians Research Network (KDRN). The survey comprised 44 questions (Appendix 1) including questions regarding the number of services offering KD therapy, number and age of patients treated, waiting time to commence $K D$, duration on $K D$ and types of $K D$ in use. The survey was circulated 
via the KDRN and Ketogenic Professional Advisory Group (KetoPAG) mailing lists and advertised through the parental support charities, Matthew's Friends and The Daisy Garland. Each service was asked to complete only one survey. Data were collected in July 2017 and approved by the audit and research departments of individual centres as required. The results were compared with the findings of earlier surveys published in $2000^{(18)}$ and $2010^{(19) .}$

\section{Results}

\section{Demographics}

Twenty-five KD services across the UK and Ireland completed the full survey. An additional $14 \mathrm{KD}$ services provided data on the number of patients being treated with $\mathrm{KD}$ and those waiting to commence treatment but declined or were unable to complete the full survey for unreported reasons.

\section{Ketogenic diet services}

The number of services offering KDs for the treatment of epilepsy grew from 22 in 2000, (18) to 28 in $201{ }^{(19)}$, to 39 in 2017 representing a 77\% increase (Figure 1).

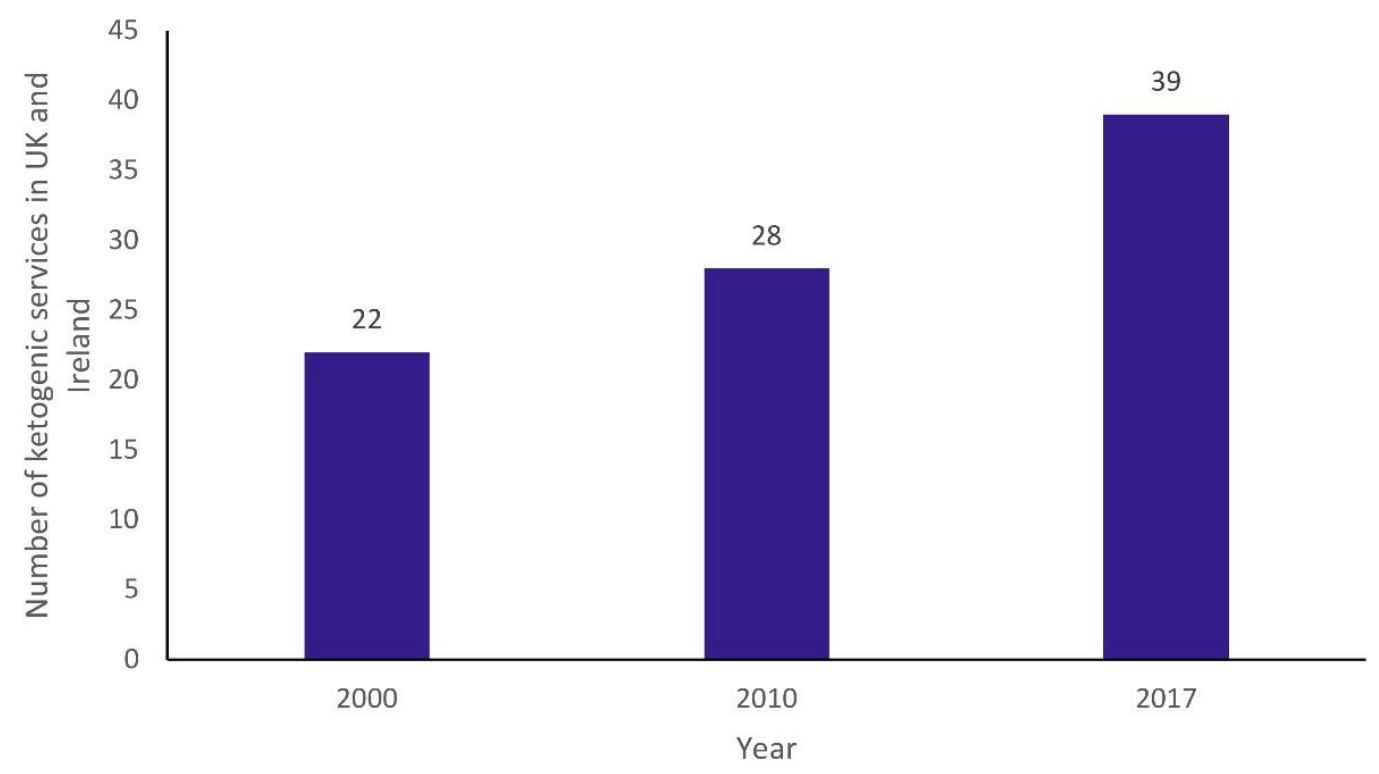

Figure 2 shows the 39 centres in the UK and Ireland offering a service in 2017, with comparative size and patient group (paediatric, adult or both) treated. Two services offered KD therapy to adults in $201{ }^{(19)}$, compared with seven in 2017. 


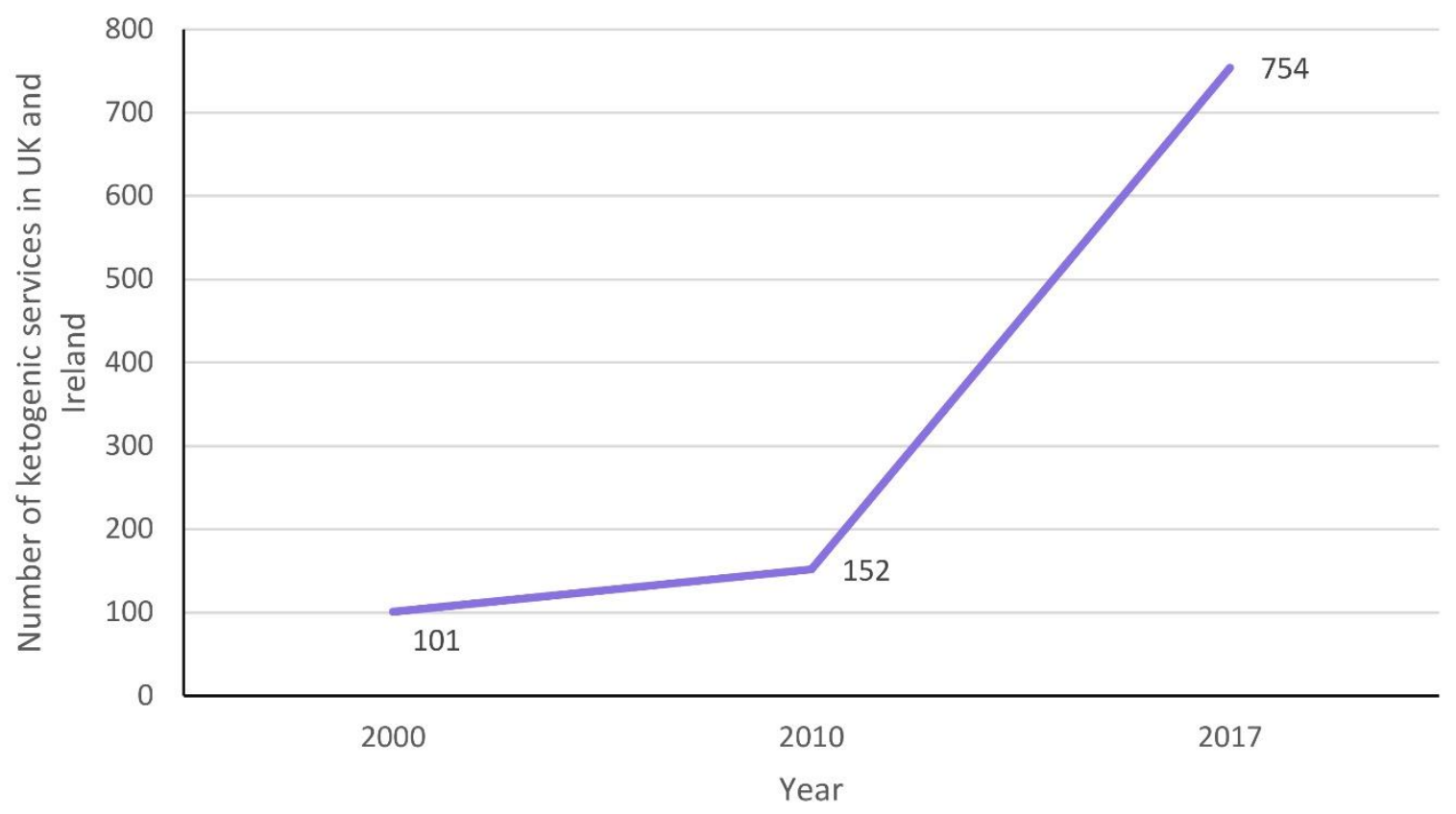

\section{Types of KDs used}

Twenty-six (of 39) ketogenic services responded to this section of the survey. $324 / 580$ (55.9\%) patients were following a classical KD, 187/580 (32.2\%) MKD, $56 / 580$ (9.7\%) MCT KD and 13/580 (2.2\%) LGIT (Figure 4a). The classical and MCT KDs were the only diets in use in $2000{ }^{(18)}$ and $2010^{(19) .}$

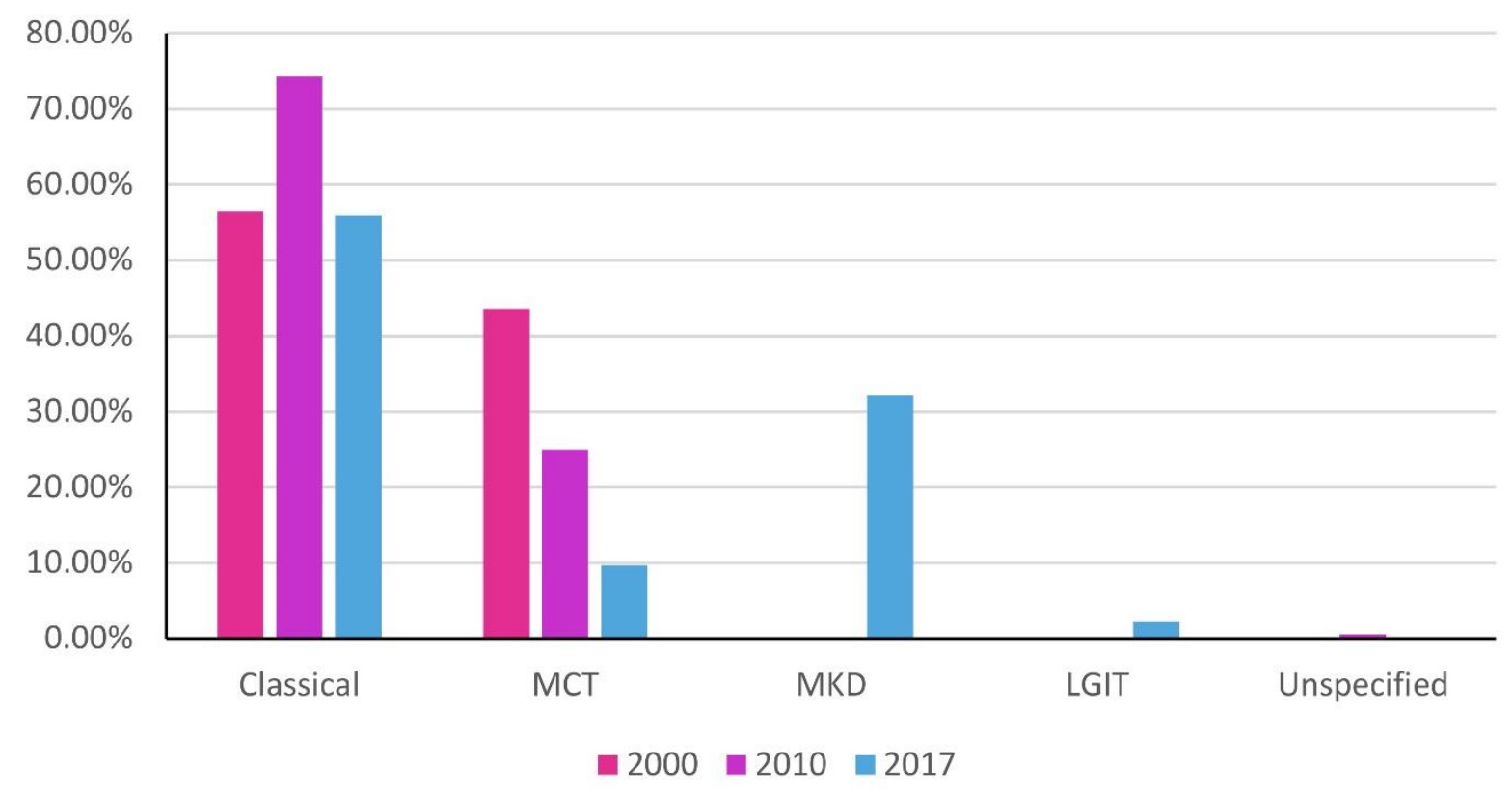

In 2017, of the 324 patients following a classical KD, 142 (43.8\%) were orally fed, 36 $(11.2 \%)$ were enterally and orally-fed, and $146(45 \%)$ were fully enterally-fed. Of the 187 patients following MKD, 183 (97.9\%) patients fed orally, 3 (1.6\%) were both 
enterally and orally-fed, and $1(0.5 \%)$ were fully enterally-fed. Of the 56 patients following MCT KD, 40 (71.4\%) fed orally, 8 (14.2\%) were enterally and orally-fed, and $8(14.2 \%)$ were fully enterally-fed. 13 (100\%) patients were following a LGIT orally. No service reported use of the MAD (Figure $4 b$ ).

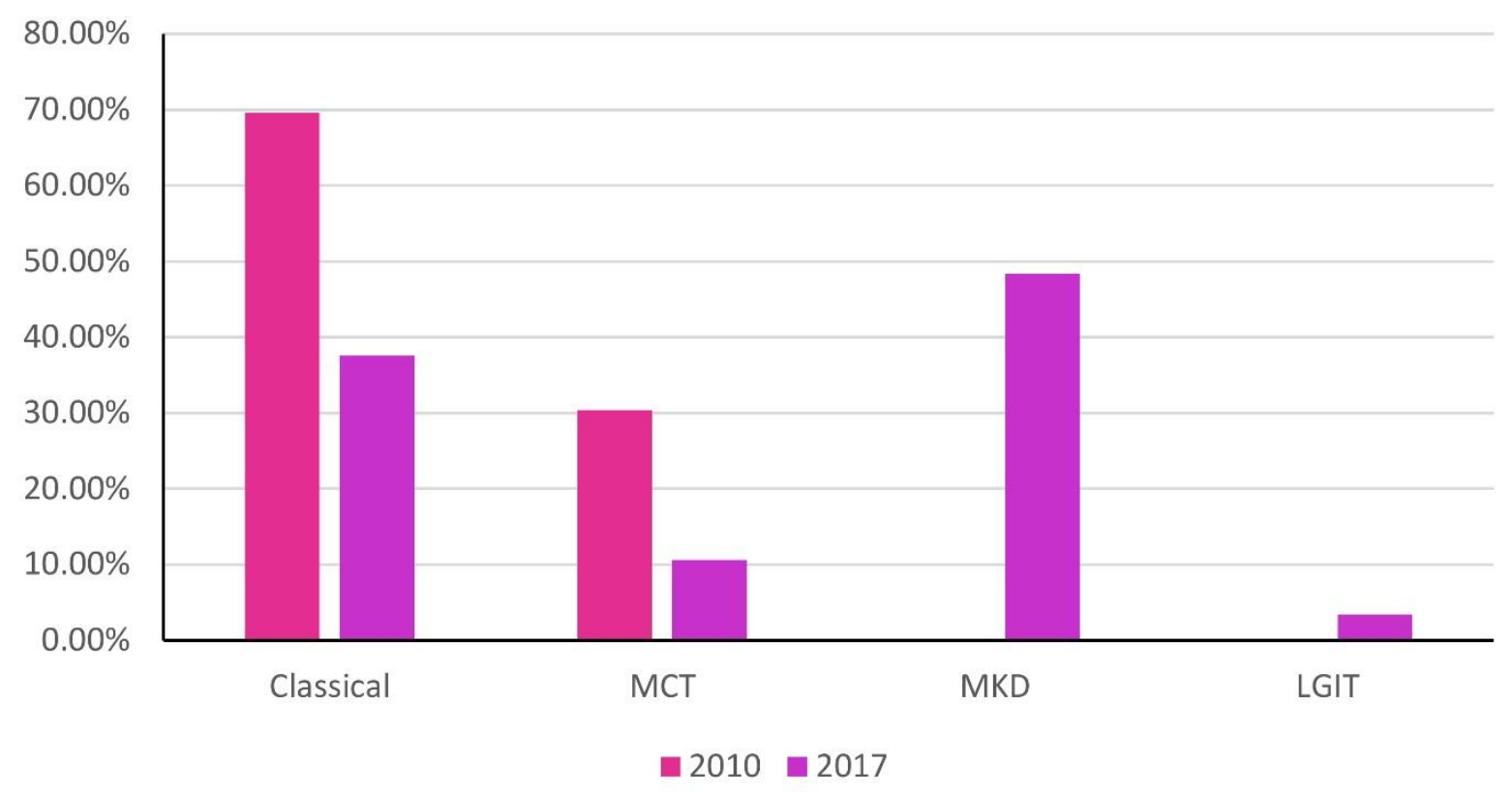

\section{$K D$ initiation}

$180 / 580(31 \%)$ of patients commenced a KD in the acute hospital setting which is similar to $43 / 132(33 \%)$ of patients in $201{ }^{(19)}$ and $52 / 101(51 \%)$ in $2000^{(18)}$. The remainder commenced a KD at home as an outpatient.

\section{KD service provision}

$7 / 26(27 \%)$ of ketogenic services were supported by charity funding, with the remainder funded predominantly by the heath service. Almost half of all ketogenic services reported inadequate funding for dietitians 11/26 (42\%), with some centres reporting inadequate funding for nurses $3 / 26$ (12\%) and consultants 2/26 (8\%) which has limited service provision for new patients. 176/580 (30.3\%) patients were treated with a KD for over 2 years and 4 services stated that this extended use of KD treatment, limited their service provision for new patients. Only 5 services had access to a transition service for children $\geq 18$ years; the shortage of transition services also limited ketogenic service provision. 


\section{Discussion}

The number of KD services has increased by over two thirds since 2000, with a concomitant increase of $647 \%$ in patient numbers. This is likely due, in part, to the growing body of evidence supporting use of the KD for epilepsy ${ }^{(8)}$ and the inclusion of KDs in NICE guidance ${ }^{(20)}$. A similar study of French KD services ${ }^{(21)}$ also reports an increase in the number of KD services and patients treated from 2005 to 2018.

Use of the classical KD has reduced substantially since 2000 , predominantly replaced with the MKD, which almost half of all orally-fed patients in this survey are following. Despite the lack of published evidence for the effectiveness of MKD for epilepsy, use of this KD variant may have increased as it is viewed as easier to implement by dietitians, reducing the dietetic time required to calculate the dietary prescription and to educate patients ${ }^{(8)}$. The less restrictive MKD may be better tolerated and have higher compliance levels compared with the classical and MCT $\mathrm{KDs}$, but further research is needed to support this and to determine its efficacy for epilepsy.

In 2000, it was common practice to initiate KD in the acute hospital setting ${ }^{(18)}$ and the original International Consensus Statement (2009) (22) on Optimal Clinical Management of KDs in epilepsy supported this practice. In $2010{ }^{(19)}$, half of all responding centres initiated KDs on an outpatient basis. This trend continued in our survey, with the majority of ketogenic services in the UK and Ireland favouring outpatient KD initiation. The patients who commenced KD in the acute setting may have been admitted for other reasons, including poor epilepsy control rather than exclusively to start a KD. Commencing a KD as an outpatient is generally less resource intensive for the service and is likely to be less of a burden for families.

Although not yet recommended as part of current NICE guidance, there has been an increase in availability of adult services. Research into KD use in this patient group increases $(16,17,24)$, although studies are limited by small sample sizes and there remains a need for a large scale RCT exploring clinical and cost effectiveness. Our survey has highlighted that a third of patients on a KD remain on diet over the recommended two year treatment period ${ }^{(23)}$, which could contribute to the greater 
demand for adult services. Disappointingly, despite the introduction of some adult services, only $5 / 26(19 \%)$ of paediatric centres stated they had access to an adult or transition service to refer their older patients ( $\geq 18$ years) to. This is an ongoing challenge for the managing paediatric ketogenic team but more significantly, the family who may have to consider weaning their child from KD, even when a positive response is still maintained.

The present study has some limitations. The survey was not validated and could be subject to reporter bias. Whilst, this was a practical and cost-efficient method of contacting nationwide ketogenic services, missing or incomplete data are inevitable, and the time restraints of clinical practice may have limited the depth of response on some occasions. On analysing the data this provided more questions; how long patients are waiting to access KD services, why are patients staying on diet over 2 years and what service adjustments or improvements are being implemented to reduce waiting lists or waiting times.

In conclusion, there is ongoing demand for KD for both paediatric and adult patients. Despite the rapid increase in services and increased availability of the diet, there are still substantial waiting lists, highlighting the need for continued expansion of services nationally. With almost $80 \%$ of services stating that the service size is limited by funding, new models of service delivery and funding need to be found to ensure that patients can access an effective treatment in a timely manner.

\section{References}

1. Wilder RM. The effects of ketonemia on the course of epilepsy. Mayo Clin Proceed. 1921;2:2.

2. Neal EG, Chaffe H, Schwartz RH et al. The ketogenic diet for the treatment of childhood epilepsy: a randomised controlled trial. Lancet Neurol. 2008;7:500-506.

3. Huttenlocher PR, Wilbourn AJ, Signore JM. Medium-chain triglycerides as a therapy for intractable childhood epilepsy. Neurology. 1971;21:1097-1103.

4. Kossoff EH, Cervenka MC, Henry BJ et al. A decade of the modified Atkins diet (2003-2013): Results, insights, and future directions. Epilepsy Behav. 2013;29:437-442. 
5. Martin-McGill KJ, Marson AG, Tudur Smith $C$ et al. Ketogenic diets as an adjuvant therapy in glioblastoma (the KEATING trial): study protocol for a randomised pilot study. Pilot Feasability Stud. 2017;3:67.

6. Martin-McGill KJ, Lambert B, Whitelely VJ et al. Understanding the core principles of a 'modified ketogenic diet': a UK and Ireland perspective. J Hum Nutr Diet. 2019. https://doi.org/10.1111/jhn.12637

7. Pfeifer HH, Thiele EA. Low-glycemic-index treatment: a liberalized ketogenic diet for treatment of intractable epilepsy. Neurology. 2005;65:1810-2.

8. Martin-Mcgill KJ, Jackson CF, Breshnahan R et al. Ketogenic diet and other dietary treatments for epilepsy. Cochrane Database of Syst Rev. 2018. Art. No.: CD001903

9. Neal EG, Chaffe $\mathrm{H}$, Schwartz $\mathrm{RH}$ et al. A randomized trial of classical and medium-chain triglyceride ketogenic diets in the treatment of childhood epilepsy. Epilepsia. 2009;50:1109-1117.

10. Seo JH, Lee YM, Lee JS et al. Efficacy and tolerability of the ketogenic diet according to lipid:nonlipid ratios-comparison of 3:1 with 4:1 diet. Epilepsia. 2007;48:801-805.

11. Kim JA, Yoon JR, Lee EJ et al. Efficacy of the classic ketogenic and the modified Atkins diets in refractory childhood epilepsy. Epilepsia. 2016;57:51-58.

12. Kossoff EH, Turner Z, Bluml RM et al. A randomized, crossover comparison of daily carbohydrate limits using the modified Atkins diet. Epilepsy Behav. 2007;10:432-436.

13. Martin-McGill KJ, Jenkinson MD, Tudur Smith $C$ et al. The modified ketogenic diet for adults with refractory epilepsy: An evaluation of a set up service. Seizure. 2017;52:1-6.

14. Schoeler NE, Wood S, Aldridge $V$ et al. Ketogenic dietary therapies for adults with epilepsy: Feasibility and classification of response. Epilepsy Behav. 2014;37:7781.

15. Nei M, Ngo L, Sirven Jl et al. Ketogenic diet in adolescents and adults with epilepsy. Seizure. 2014;23:439-442.

16. Zare M, Okhovat AA, Esmaillzadeh $A$ et al. Modified Atkins diet in adult with refractory epilepsy: a controlled randomized clinical trial Iran J Neurol. 2017;16:7277.

17. Kverneland M, Molteberg E, Iversen $\mathrm{PO}$ et al. Effect of modified Atkins diet in adults with drug-resistant focal epilepsy: A randomized clinical trial. Epilepsia. 2018;59:1567-1576.

18. Magrath G, MacDonald A, Whitehouse W. Dietary practices and use of the ketogenic diet in the UK. Seizure. 2000;9:128-30.

19. Lord K, Magrath G. Use of the ketogenic diet and dietary practices in the UK. J Hum Nutr Diet. 2010;23:126-132.

20. National Institute for Health and Care Excellence (NICE). Epilepsies: diagnosis and management CG137. 2012.

21. Dozieres-Puyravel B, Francois L, de Lucia S et al. Ketogenic diet therapies in France: State of the use in 2018. Epilepsy Behav. 2018;86:204-206.

22. Kossoff EH, Zupec-Kania BA, Amark PE et al. Optimal clinical management of children receiving the ketogenic diet: Recommendations of the International Ketogenic Diet Study Group. Epilepsia. 2009;50:304-17. 
23. Kossoff EH, Zupec-Kania BA, Auvin S et al. Optimal clinical management of children receiving dietary therapies for epilepsy: Updated recommendations of the International Ketogenic Diet Study Group. Epilepsia Open. 2018;3:175-92.

24. McDonald TJW, Cervenka MC. Ketogenic diets for adult neurological disorders. Neurtherapeutics. 2018;15:1018-1031. 